\title{
STABILITY OF PERIODIC ORBITS IN THE THEOREM OF ŠARKOVSKII
}

\author{
LOUIS BLOCK
}

\begin{abstract}
Let $f$ be a continuous map of a closed, bounded interval into itself. It is shown that the conclusion of the theorem of Sarkovskii holds for perturbations of $f$. In other words, if $f$ has a periodic point of period $k$, and $g$ is a continuous map close to $f$, then $g$ has periodic points of certain periods.
\end{abstract}

1. Introduction. This paper is concerned with the periodic orbits of continuous mappings of the interval to itself. Such mappings (sometimes called first order difference equations when studied from this point of view) arise as models for various phenomena in the natural sciences. See [2] or [3] for some examples and references.

Let $I$ denote a closed, bounded, interval on the real line, and let $C^{0}(I, I)$ denote the space of continuous mappings of $I$ into itself with the topology of uniform convergence. For $f \in C^{0}(I, I)$ let $P(f)$ denote the set of positive integers $k$ such that $f$ has a periodic point of least period $k$.

Consider the following ordering of the positive integers:

$$
\begin{aligned}
1,2,4,8, \ldots . & \ldots 7 \cdot 8,5 \cdot 8,3 \cdot 8, \ldots, \\
& 7 \cdot 4,5 \cdot 4,3 \cdot 4, \ldots, 7 \cdot 2,5 \cdot 2,3 \cdot 2, \ldots, 7,5,3 .
\end{aligned}
$$

We will refer to this ordering as the Šarkovskii ordering. A. N. Šarkovskii has proved the following theorem (see [4] or [5]).

TheOrem (ŚSARKovSKi). Let $f \in C^{0}(I, I)$. If $n \in P(f)$ and $k$ is to the left of $n$ in the Sarkovskii ordering then $k \in P(f)$.

In studying properties of the orbits of mappings, it is desirable to prove that a given property is stable, in the sense that if a map $f$ has the property then perturbations of $f$ (maps close to $f$ in an appropriate topological space) also have the given property. This is important from a physical point of view, because when one uses a mapping as a model of some phenomena there will normally be some possibility for error. For most stability theorems, one must consider $C^{1}$ mappings with some restrictions and $C^{1}$ perturbations. However, in this paper we prove the following result, which shows that some stability exists in Šarkovskii's Theorem, in the $C^{0}$ topology for all $f \in C^{0}(I, I)$.

Received by the editors November 2, 1979 and, in revised form, February 24, 1980; presented to the Society, January 3, 1980.

1980 Mathematics Subject Classification. Primary 54H20.

Key words and phrases. Periodic point, period of a periodic point.

(C) 1981 American Mathematical Society 0002-9939/81/0000-0088/\$02.00 
MAIN TheORem. Let $f \in C^{0}(I, I)$ and suppose that $n \in P(f)$. There is a neighborhood $N$ of $f$ in $C^{0}(I, I)$ such that for every $g \in N$ and every positive integer $k$ with $k$ to the left of $n$ in the Sarkovskii ordering, $k \in P(g)$.

In proving this result, we use a theorem of Štefan, [5], and a lemma from [1] (both of which will be stated in the next section), in addition to the theorem of Sarkovskii.

We remark that in general if $f \in C^{0}(I, I)$ (or $f \in C^{1}(I, I)$ ) and $n \in P(f)$ then perturbations of $f$ need not have a periodic point of period $n$.

2. Preliminary definitions and results. Let $f \in C^{0}(I, I)$. For any nonnegative integer $n$, we define $f^{n}$ inductively by $f^{0}$ is the identity map of $I$ and $f^{n}=f \circ f^{n-1}$.

Let $p \in I$. We say $p$ is a periodic point of $f$ if $p$ is a fixed point of $f^{n}$ for some positive integer $n$. If $p$ is a periodic point of $f$, the smallest positive integer $n$ with $f^{n}(p)=p$ is called the period of $p$.

We define the orbit of $p$ to be $\left\{f^{n}(p): n=0,1,2, \ldots\right\}$. If $p$ is a periodic point, we say the orbit of $p$ is a periodic orbit, and we define the period of the orbit to be the period of $p$.

Finally, for $f \in C^{0}(I, I)$ we let $P(f)$ denote the set of positive integers $k$ such that $f$ has a periodic point of period $k$.

We now state two elementary lemmas which will be used in the next section.

LeMma 1. Let $f \in C^{0}(I, I)$. Suppose $M$ and L are closed subintervals of $I$ such that $f(M) \supset L$. Then there is a closed interval $K \subset M$ with $f(K)=L$.

Lemma 2. Let $f \in C^{0}(I, I)$. Suppose $K$ is a closed subinterval of $I$ with $K \subset f(K)$. Then $f$ has a fixed point in $K$.

We will also use the following lemma from [1] (see Lemma 10 in $\$ 3$ of [1]).

Lemma 3. Let $f \in C^{0}(I, I)$. Suppose $f$ has a periodic orbit $\left\{p_{1}, p_{2}, p_{3}, p_{4}\right\}$ of period 4 with $p_{1}<p_{2}<p_{3}<p_{4}$. If $f\left(\left\{p_{1}, p_{2}\right\}\right) \neq\left\{p_{3}, p_{4}\right\}$ then $f$ has a periodic point of period 3.

We conclude this section by stating the following theorem of Stefan (see Theorem 2 of [5]) which will be used in the next section.

Theorem ('̌́tefan). Let $f \in C^{0}(I, I)$. Suppose $n \in P(f)$ where $n$ is odd and $n>1$, but $j \notin P(f)$ for all $j \in\{3,5, \ldots, n-2\}$. Let $\left\{p_{1}, \ldots, p_{n}\right\}$ be a periodic orbit of $f$ of period $n$ with $p_{1}<p_{2}<\cdots<p_{n}$. Let $t=(n+1) / 2$. Then either (A) or (B) holds.

(A) $f\left(p_{t-k}\right)=p_{t+k}$ for $k=1, \ldots, t-1, f\left(p_{t+k}\right)=p_{t-k-1}$ for $k=0, \ldots, t-2$, and $f\left(p_{n}\right)=p_{t}$.

(B) $f\left(p_{t-k}\right)=p_{t+k+1}$ for $k=0, \ldots, t-2, f\left(p_{t+k}\right)=p_{t-k}$ for $k=1, \ldots, t-1$, and $f\left(p_{1}\right)=p_{t}$.

\section{Proof of the Main Theorem.}

LeMma 4. Let $f \in C^{0}(I, I)$ and let $k$ be an odd positive integer with $k>3$. Suppose that there is a point $y \in I$ such that the following hold. 
(1) $f^{k-2}(y)<f^{k-4}(y)<f^{k-6}(y)<\cdots<f^{3}(y)<f(y)<y$.

(2) $y<f^{2}(y)<f^{4}(y)<f^{6}(y)<\cdots<f^{k-1}(y)$.

(3) $y<f^{k}(y)$.

Then $k \in P(f)$.

Proof. Since $f(y)<y$ and $f(f(y))>f(y), f$ has a fixed point $e \in(f(y), y)$. Let $M_{1}=[e, y], M_{3}=\left[y, f^{2}(y)\right], \ldots$, and $M_{k}=\left[f^{k-3}(y), f^{k-1}(y)\right]$. Also, let $M_{2}=$ $[f(y), e], M_{4}=\left[f^{3}(y), f(y)\right], \ldots$, and $M_{k-1}=\left[f^{k-2}(y), f^{k-4}(y)\right]$.

Note that $f\left(M_{j}\right) \supset M_{j+1}$ for each $j=1, \ldots, k-1$ and $f\left(M_{k}\right) \supset M_{1}$. Hence, by Lemma 1 , there are closed intervals $K_{1}, K_{2}, \ldots, K_{k}$ with $K_{j} \subset M_{j}$ for each $j=$ $1, \ldots, k$ such that $f\left(K_{k}\right)=M_{1}$ and $f\left(K_{j}\right)=K_{j+1}$ for each $j=1, \ldots, k-1$. It follows that $f^{k}\left(K_{1}\right)=M_{1}$. By Lemma $2, f^{k}$ has a fixed point $z \in K_{1}$.

Suppose that $f^{j}(z)=z$ for some $j \in\{1, \ldots, k-1\}$. Since $f^{j}(z) \in M_{j+1}$, it follows that either $j=1$ and $z=e$, or $j=2$ and $z=y$. But $z=e$ is impossible since $f^{2}(z) \in M_{3}$. Also, $j=2$ and $z=y$ is impossible since $y<f^{2}(y)$. Hence $f^{j}(z) \neq z$ for all $j \in\{1, \ldots, k-1\}$. Thus, $z$ is a periodic point of $f$ of period $k$.

Lemma 5. Let $f \in C^{0}(I, I)$ and suppose $n \in P(f)$ where $n$ is odd and $n>3$. Then there is a neighborhood $N$ of $f$ in $C^{0}(I, I)$ with the property that if $g \in N$ then $(n+2) \in P(g)$.

Proof. By the theorem of Šarkovskii it suffices to prove this lemma in the case that $j \notin P(f)$ for all $j \in\{3,5, \ldots, n-2\}$. Hence, we assume that this is the case.

Since $n \in P(f), f$ has a periodic orbit $\left\{p_{1}, \ldots, p_{n}\right\}$ of period $n$, with $p_{1}<p_{2}$ $<\cdots<p_{n}$. Either (A) or (B) (of the theorem of Stefan) must hold. Since these are analogous we may assume that $(\mathrm{A})$ holds.

Let $t=(n+1) / 2$ and let $z=p_{t}$. Then

(1) $f^{n-2}(z)<f^{n-4}(z)<f^{n-6}(z)<\cdots<f^{3}(z)<f(z)<z$.

(2) $z<f^{2}(z)<f^{4}(z)<f^{6}(z)<\cdots<f^{n-1}(z)$.

(3) $z=f^{n}(z)$.

Now, since $f(f(z))>z$ and $f(z)<z$, there is a point $b \in(f(z), z)$ with $f(b)=z$. Also, since $f(b)>b$ and $f(z)<b$, there is a point $y \in(b, z)$ with $f(y)=b$. Thus, $f^{2}(y)=z$. It follows that $y$ satisfies the conditions of Lemma 4 with $k=n+2$. Thus there is a neighborhood $N$ of $f$ such that $y$ satisfies the conditions of Lemma 4 with $k=n+2$ for all $g \in N$. Therefore $n+2 \in P(g)$ for all $g \in N$.

Lemma 6. Let $f \in C^{0}(I, I)$. Suppose $n \in P(f)$ and $n=r \cdot s$ where $r=2^{i}$ for some nonnegative integer $i$, and $s$ is an odd integer with $s>3$. Then there is a neighborhood $N$ of $f$ in $C^{0}(I, I)$ such that for every $g \in N$, and every positive integer $k$ with $k$ to the left of $n$ in the Śarkovskii ordering, $k \in P(g)$.

Proof. Note that $n \in P(f)$ implies that $s \in P\left(f^{r}\right)$. By Lemma 5, there is a neighborhood $N_{1}$ of $f^{r}$ with the property that if $g \in N_{1}$ then $(s+2) \in P(g)$.

Since the mapping $g$ to $g^{r}$ is continuous, there is a neighborhood $N$ of $f$ such that if $g \in N$ then $g^{r} \in N_{1}$.

Let $g \in N$. Then $g^{r} \in N_{1}$. Hence, $(s+2) \in P\left(g^{r}\right)$. This implies that $(s+2) \cdot j$ $\in P(g)$, where $j=2^{t}$ for some integer $t$ with $0<t<i$. It follows from the theorem 
of Sarkovskii that for every positive integer $k$ with $k$ to the left of $n$ in the Sarkovskii ordering, $k \in P(g)$.

Lemma 7. Let $f \in C^{0}(I, I)$ and suppose $4 \in P(f)$. Then there is a neighborhood $N$ of $f$ in $C^{0}(I, I)$ such that if $g \in N$ then $2 \in P(g)$.

Proof. By hypothesis $f$ has a periodic orbit $\left\{p_{1}, p_{2}, p_{3}, p_{4}\right\}$ of period 4 with $p_{1}<p_{2}<p_{3}<p_{4}$. If $3 \in P(f)$ then the conclusion of this lemma follows from Lemma 6. Hence, we may assume that $3 \notin P(f)$. This implies, by Lemma 3 , that $f\left(\left\{p_{1}, p_{2}\right\}\right)=\left\{p_{3}, p_{4}\right\}$. Hence, we must also have $f\left(\left\{p_{3}, p_{4}\right\}\right)=\left\{p_{1}, p_{2}\right\}$. Thus, $f\left(\left[p_{1}, p_{2}\right]\right) \supset\left[p_{3}, p_{4}\right]$ and $f\left(\left[p_{3}, p_{4}\right]\right) \supset\left[p_{1}, p_{2}\right]$.

By Lemma 1, there is a closed interval $K \subset\left[p_{3}, p_{4}\right]$ with $f(K)=\left[p_{1}, p_{2}\right]$. Let $v$ be the element of $\left\{p_{1}, p_{2}\right\}$ with $f(v)=p_{4}$. For some $x \in K, f(x)=v$. Since $x \neq p_{4}$, $f^{2}(x)>x$. Similarly $f^{2}(y)<y$ for some $y \in K$. There is a neighborhood $N$ of $f$ such that if $g \in N$, then $g^{2}(x)>x, g^{2}(y)<y$ and $g(z)<p_{3}$ for all $z \in K$. Then $2 \in P(g)$ for all $g \in N$.

Lemma 8. Let $f \in C^{0}(I, I)$. Suppose $2^{m} \in P(f)$ for some positive integer $m$. Then there is a neighborhood $N$ of $f$ in $C^{0}(I, I)$ such that if $g \in N$ then $2^{i} \in P(g)$ for every integer $i$ with $0 \leqslant i \leqslant m-1$.

Proof. We may assume $m \geqslant 2$. Let $r=2^{m-2}$. Then $4 \in P\left(f^{r}\right)$. By Lemma 7, $2 \in P(g)$ for every $g$ in some neighborhood $N_{1}$ of $f^{r}$. There is a neighborhood $N$ of $f$ such that if $g \in N$ then $g^{r} \in N_{1}$.

Let $g \in N$. Then since $2 \in P\left(g^{r}\right), 2^{m-1} \in P(g)$. By the theorem of Šarkovskii, it follows that $2^{i} \in P(g)$ for every integer $i$ with $0<i<m-1$.

The Main Theorem follows immediately from Lemma 6 and Lemma 8.

\section{REFERENCES}

1. L. Block, Simple periodic orbits of mappings of the interval, Trans. Amer. Math. Soc. 254 (1979), 391-398.

2. T. Li and J. A. Yorke, Period three implies chaos, Amer. Math. Monthly 82 (1975), 985-992.

3. R. M. May, Simple mathematical models with very complicated dynamics, Nature 261 (1976), 459-467.

4. A. N. Sarkovskii, Coexistence of cycles of a continuous map of a line into itself, Ukrain. Mat. Ż. 16 (1964), 61-71.

5. P. Stefan, A theorem of Sarkouskii on the existence of periodic orbits of continuous endomorphisms of the real line, Comm. Math. Phys. 54 (1977), 237-248.

Department of Mathematics, University of Florida, Ganesville, Florida 32611 